\section{PTH-029 UGI CANCERS - ARE WE LOOKING?}

Vivek Goodoory*, Lian Hodges, Benjamin Crooks, Mark Murgatroyd, Helen White, Tom Butler, Javaid Iqbal. Manchester University NHS Foundation Trust, Manchester, UK

\subsection{6/gutjnl-2018-BSGAbstracts.50}

Introduction Oesophago-gastric (OG) cancers in the UK often present at an advanced stage, and hence reduced chance of curative therapy. A recent meta-analysis involving 3787 patients with OG cancer has shown that $11.3 \%$ OG cancers are missed at endoscopy 3 years before diagnosis. Recent guidelines from the BSG recommend that endoscopy units should audit for potential missed pathology in those diagnosed who have undergone an endoscopy in the preceding 3 years.

Methods This was a retrospective audit at a regional upper gastrointestinal centre reviewing all cases presenting with OG cancer over a 2 year period between Sept 2015 and Sept 2017. Data was collected from the electronic database, case notes and the GI reporting tool, in all patients to ascertain if an OGD was performed within 3 years prior to diagnosis. This included site of cancer, stage, endoscopist, probable reason for missed pathology and assessment of certain KPI's pertaining to the endoscopy.

Results 105 patients were diagnosed with OG cancers during this period. Median age 74 years; M:F 69:31; Oesophageal 60\%:stomach $40 \%$. Twenty-two patients (21\%) had an OGD in the 3 years prior to their index (diagnostic) OGD; 11 $(10.5 \%)$ were deemed 'not missed' cancers because there were valid reasons for repeating an endoscopy; and 11 (10.5\%) were thought to represent 'missed' opportunities of diagnosing cancer in the preceding 3 years. The median time interval between the 1 st OGD and index OGD were 20 and 270 days for 'not-missed' and 'missed' groups respectively. Possible reasons for missed cancer were lack of lesion recognition (5/ $46 \%)$, dual pathology (1/9\%), technical limitations of OGD $(1 / 9 \%)$ or a combination of factors $(4 / 36 \%)$. Adequacy of mucosal visualisation was not photo-documented in $64 \%$ of cases. The main reason for early repeat endoscopy in the 'non-missed' group was a high index of suspicion of pathology on initial OGD without any histological confirmation.

Conclusions A missed cancer rate of $10.5 \%$ in a regional upper GI centre is similar to published rates in a recent metaanalysis but does not achieve the minimal standard of $<10 \%$ set by the BSG. These results were discussed at the EUGM and various measures being undertaken to reduce this include: modifications to optimise visualisation (simethicone pre-procedure), rigorous photo-documentation, dedicated surveillance lists eg. Barretts. This will be re-audited in 3 years.

\section{PTH-030 CONVENTIONAL VERSUS VIRTUAL CHROMOENDOSCOPY FOR COLITIS SURVEILLANCE: DYSPLASIA DETECTION, FEASIBILITY AND PATIENT ACCEPTABILITY (CONVINCE)}

\begin{abstract}
${ }^{1}$ Shraddha Gulati*, ${ }^{1}$ Patrick Dubois, ${ }^{2}$ Ben Carter, ${ }^{3}$ Victoria Cornelius, ${ }^{1}$ Andrew Emmanuel, ${ }^{1}$ Amyn Haji, ${ }^{1}$ Bu'Hussain Hayee. ${ }^{1}$ King's College Hospital, London, UK; ${ }^{2}$ King's College London, London, UK; ${ }^{3}$ Imperial College, UK
\end{abstract}

\subsection{6/gutjnl-2018-BSGAbstracts.51}

Introduction Chromoendoscopy (CE) is the recommended surveillance technique for dysplasia in colitis, but uptake has been limited. Virtual CE (VCE) by Fujinon Intelligent Colour Enhancement digitally reconstructs mucosal images in real- time, without the technical challenges of CE. The literature provides limited information on patient experience (PE); imperative to adherence to surveillance programmes. We performed a multi-faceted randomised crossover trial to evaluate acceptability of study design and obtain preliminary comparative procedural performance data and PE using CE vs VCE.

Methods Patients 18-75 y due surveillance colonoscopy were randomised to undergo CE or VCE first. After 3-8 weeks, participants underwent colonoscopy with the second technique, performed by an endoscopist blinded to the results of the first. Patient recruitment/retention, missed dysplasia by VCE/CE, endoscopist's prediction of dysplasia and contamination (endoscopists memory/sampling of the 1 st procedure) were recorded. PE was assessed by validated questionnaires. This abstract presents independent research funded by the NIHR under its Research for Patient Benefit Programme (PBPG-0614-34040). The views expressed are those of the authors and not necessarily those of the NHS, the NIHR or the Department of Health.

Results 60 patients were recruited (recruitment rate $80 \%$ ) over an 11 month period. 48 patients completed the trial (retention rate $80 \%) ; 23(11 \mathrm{~F}, 48.4 \pm 14.6 \mathrm{y})$ received $\mathrm{VCE}$ and 25 (9 F,41.4 \pm 12.3 y) CE first. Examination time for CE vs VCE was $20 \pm 7$ vs $14 \pm 4$ mins respectively $(p<0.001$; CI $3.5-8)$. There were no episodes of contamination. 11 dysplastic lesions were detected in $7 / 48$ (14.5\%). Per-lesion analysis: VCE missed 1 lesion (miss rate 9.1\%), CE missed 2 lesions in 2 patients (miss rate $18.2 \%$ ). Per-patient analysis: miss rate for dysplasia using VCE was 1/48 (2.1\%) VCE and CE 2/48 (4.2\%). Diagnostic accuracy for dysplasia using VCE 93.94\% (85.2-98.32) vs CE 76.9\% (66.9\%-98.2\%). Visual analogue scale for pain experienced using VCE and CE were 27.4 $\pm 17.5 \mathrm{~mm}$ and $34.7 \pm 18 \mathrm{~mm}$ respectively. Patient preference for VCE was $67 \%(n=31)$ vs CE $33 \%(n=15)$ in $n=46$, $\mathrm{p}<0.001$.

Conclusions This is the first study to incorporate PE in a colitis surveillance trial and has demonstrated feasibility of the trial design itself. VCE is safe, appears technically less challenging, quicker and more comfortable procedure for patients with dysplasia detection at least as good as CE, thus overcoming many of barriers to the wider adoption of CE. This trial forms the successful foundation to inform a multicenter trial to confirm the value of VCE for colitis surveillance.

\section{PTH-031 THE OUTCOMES OF ERCP FOR COMMON BILE DUCT GALLSTONES IN ENGLAND BETWEEN 2003 AND 2015}

${ }^{1}$ Philip Harvey*, ${ }^{2}$ Simon Baldwin, ${ }^{2}$ Jemma Mytton, ${ }^{2}$ Ben Coupland, ${ }^{2}$ Felicity Evison, ${ }^{2}$ Prashant Patel, ${ }^{1}$ Nigel Trudgill. 'Sandwell And West Birmingham Nhs Trust, Birmingham, UK; ${ }^{2}$ University Hospital Birmingham, Birmingham, UK

\subsection{6/gutjnl-2018-BSGAbstracts.52}

Introduction The BSG ERCP standards suggest duct clearance should be achieved in $>85 \%$ of ERCPs for common bile duct gallstones. However recent data on ERCP for palliation of malignant biliary obstruction demonstrated significant variation in outcomes between providers related to procedure volume. We have therefore examined outcomes of ERCPs for common bile duct gallstones.

Methods Hospital Episode Statistics (HES) include diagnostic and procedural data for all hospital attendances in England. All subjects undergoing their first ERCP, with the ICD10 code K80 (cholelithiasis) were included. Subjects with a relevant 